\title{
Fast Navigation in a Large Hilbert Space Using Quantum Optimal Control
}

\author{
Arthur Larrouy, ${ }^{1, \dagger}$ Sabrina Patsch $\odot,{ }^{2, \dagger}$ Rémi Richaud, ${ }^{1}$ Jean-Michel Raimond $\odot,{ }^{1}$ Michel Brune $\odot,{ }^{1}$ \\ Christiane P. Koch $\oplus^{2,3}$ and Sébastien Gleyzes $\oplus^{1, *}$ \\ ${ }^{1}$ Laboratoire Kastler Brossel, Collège de France, CNRS, ENS-Université PSL, Sorbonne Université, \\ 11, place Marcelin Berthelot, 75005 Paris, France \\ ${ }^{2}$ Theoretische Physik, Universität Kassel, Heinrich-Plett-Straße 40, 34132 Kassel, Germany \\ ${ }^{3}$ Dahlem Center for Complex Quantum Systems and Fachbereich Physik, Freie Universität Berlin, \\ Arnimallee 14, 14195 Berlin, Germany
}

(Received 20 January 2020; revised manuscript received 19 March 2020; accepted 15 April 2020; published 16 June 2020)

\begin{abstract}
The precise engineering of quantum states, a basic prerequisite for technologies such as quantumenhanced sensing or quantum computing, becomes more challenging with increasing dimension of the system Hilbert space. Standard preparation techniques then require a large number of operations or slow adiabatic evolution and give access to only a limited set of states. Here, we use quantum optimal control theory to overcome this problem and derive shaped radio-frequency pulses to experimentally navigate the Stark manifold of a Rydberg atom. We demonstrate that optimal control, beyond improving the fidelity of an existing protocol, also enables us to accurately generate a nonclassical superposition state that cannot be prepared with reasonable fidelity using standard techniques. Optimal control thus substantially enlarges the range of accessible states. Our joint experimental and theoretical work establishes quantum optimal control as a key tool for quantum engineering in complex Hilbert spaces.
\end{abstract}

DOI: $10.1103 /$ PhysRevX.10.021058

\section{INTRODUCTION}

Quantum information science exploits the essential quantum features of light and matter for the design of devices with applications in computing, sensing, or communication [1]. The building blocks of any quantum device are qubits as information carriers, and their operation requires the ability to prepare, manipulate, and read out their state [2]. The simplicity of the basic concept is, however, in contrast with the complexity encountered when implementing it in an actual physical platform. Both in atom-based and solid-state devices it becomes more and more challenging to precisely control the quantum state as the size of the system increases. For example, higher-order terms of the Hamiltonian introduce nonlinearities that limit the fidelity of basic operations $[3,4]$. The complexity of the system spectrum increases, making it difficult to address individually quantum states in a short time $[5,6]$. At the

\footnotetext{
${ }^{*}$ Corresponding author. gleyzes@lkb.ens.fr

These authors contributed equally to this work.

Published by the American Physical Society under the terms of the Creative Commons Attribution 4.0 International license. Further distribution of this work must maintain attribution to the author(s) and the published article's title, journal citation, and DOI.
}

Subject Areas: Atomic and Molecular Physics,

Quantum Physics, Quantum Information same time, decoherence occurs more rapidly, which requires to operate faster [7].

Quantum optimal control $[8,9]$ is a versatile approach to address the challenge of fast navigation in a large Hilbert space. It allows one to design the time-dependent shape of experimental control knobs, electromagnetic fields, for instance, that accomplish a given task in a quantum system in the best possible way. The starting point is a performance index, such as the target state preparation fidelity, which is treated as a functional of the (yet unknown) shape of the electromagnetic control field. The essence of quantum optimal control theory is to maximize the performance index while minimizing the use of resources such as time, bandwidth, or power [8].

A large number of theoretical quantum control protocols cover most physical platforms considered for quantum technologies, notably neutral atoms [10], ions [11], photons $[12,13]$, color centers in diamond [14,15], and superconducting qubits $[16,17]$. Quantum control experiments have progressed at a much slower pace and with a focus on one or two qubits or few-level systems [18-22]. For systems with larger Hilbert spaces, such as the quantum harmonic oscillator [23,24] or an assembly of trapped atoms [7,25], optimal control approaches start to replace conventional quantum engineering designs, providing a faster alternative [26] to existing preparation protocols [27]. Here, we go further and demonstrate how quantum optimal control can be used to 
navigate a Hilbert space of large dimension, finding a strategy to reach an arbitrary quantum state, for which no intuitive preparation method is known.

Our example is the manipulation of Rydberg atoms, which represent an attractive platform for quantum technologies [28]. In particular, Rydberg atoms in long-lived circular states, where the angular momentum projection takes its maximal value, are ideal for fundamental experiments on matter-field coupling [29]. They have also been suggested for use as logical states in a quantum computer $[30,31]$ or as an optical-to-microwave interface [32,33]. Superpositions of circular states with opposite magnetic momentum or of states with very different electric dipoles are instrumental for quantum-enabled electrometers and magnetometers [4,34]. Use of circular states and their superpositions in quantum computing and sensing in particular requires the capability of fast and accurate state preparation.

In this paper, we make use of optimal control to coherently manipulate Rydberg atoms and prepare an arbitrary superposition of states in a complex Hilbert space inside the Stark manifold of rubidium. We first demonstrate a significant improvement of the preparation of the long-lived circular state in terms of fidelity and duration. Furthermore, we prepare mesoscopic superpositions of angular momentum eigenstates in the Rydberg manifold that cannot be created with standard protocols. This achievement is made possible by using quantum optimal control to tune the time-dependent phase and amplitude of the rf drive [35] that coherently manipulates the atoms inside the Rydberg manifold. Our work opens the way to generate arbitrary nonclassical superposition states in large Hilbert spaces.

\section{RYDBERG ATOMS}

We consider rubidium atoms in the presence of an electric field $\mathbf{F}$. The Hilbert space to be navigated is sketched in Fig. 1, showing the rubidium levels with a principal quantum number $n=52$ and a positive magnetic quantum number $m$. The levels are arranged in a triangular shape with the circular state $52 c$ at its tip [36]. For $m>2$, the levels are almost hydrogenic, and the transitions between nearby levels are, to first order in $F$, degenerate at the Stark frequency $\omega_{n} / 2 \pi=3 n e a_{0} F / 2 h(250 \mathrm{MHz}$ for $F=F_{0}=2.5 \mathrm{~V} / \mathrm{cm}$ ). For $m \leq 2$, the energy levels are shifted due to the electron penetration in the ionic core and the transitions can differ from a few megahertz to hundreds of megahertz with respect to $\omega_{n}$ [37].

Transitions between different $m$ sublevels couple to different polarizations of the electromagnetic field. An atom initially prepared in a state of the lowest diagonal of Fig. 1 will remain in this subspace (represented by thick lines on Fig. 1) when driven by a $\sigma^{+}$-polarized radio frequency at frequency $\omega_{\mathrm{rf}}=\omega_{n}$ (blue arrows in Fig. 1). This subspace can be described as a large spin $J=51 / 2$, evolving on a generalized Bloch sphere, with the circular state at the north pole. For the hydrogenic levels $(m>2)$,

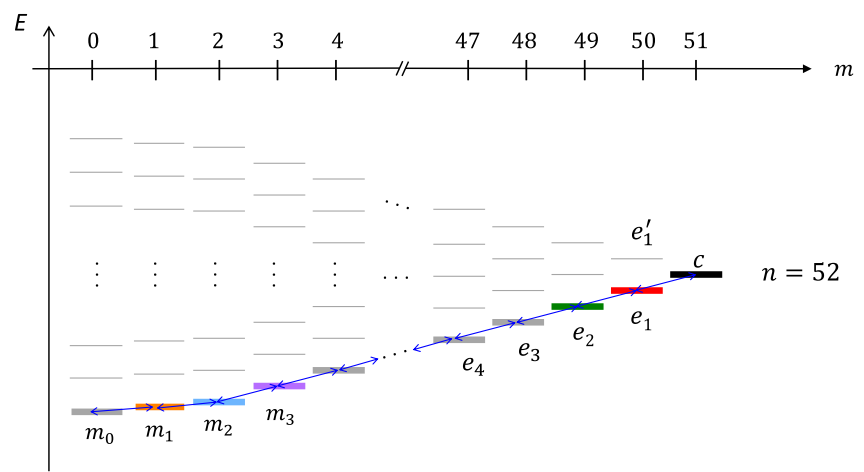

FIG. 1. Stark levels. Scheme of the energy levels for $F \sim 2.5 \mathrm{~V} / \mathrm{cm}$, sorted by $m$ values for $m \geq 0$. The circular state is labeled $c$. The levels of the lower diagonal are labeled $m_{j}$ for low- $m$ states and $e_{j}$ for high- $m$ states. Finally, $e_{1}^{\prime}$ is the Stark level with $m=50$ above the circular state. The laser excitation prepares the $m_{2}$ state. The atom is then driven by a $\sigma^{+}$rf field at frequency $\omega_{\text {rf }}=\omega_{n}$ (blue arrows). Since a $\sigma^{+}$field only couples levels with $\Delta m=+1$, the dynamics of the atom remains in the subspace of the manifold lower diagonal (levels represented as thick lines).

the rf field induces a classical rotation of the spin on this sphere [33].

The experiment takes place in a plane-parallel capacitor providing the directing field $\mathbf{F}$ aligned with the $O z$ quantization axis (Fig. 2). The atoms are prepared at $t=0$ in the lowest $m=2$ level (label $m_{2}$ on Fig. 1) by a combination of laser pulses [33] (see Appendix B). They are then manipulated by a rf field created by four electrodes circling the interval between the capacitor plates. We apply on them rf drives at $\omega_{\mathrm{rf}} / 2 \pi=250 \mathrm{MHz}$ generating near the center of the structure a $\sigma^{+}$-polarized field. After having

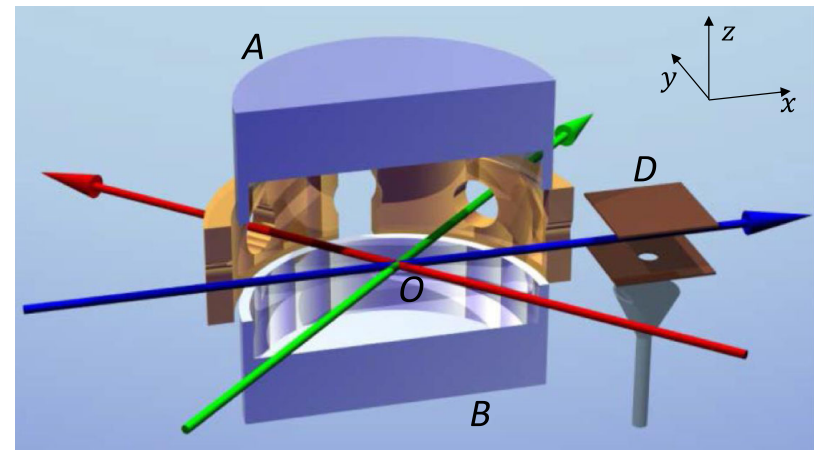

FIG. 2. Scheme of the experiment. A rubidium thermal beam (dark blue arrow), effusing from an oven, crosses a structure made of two plane-parallel electrodes $A$ and $B$ (blue) creating the vertical directing electric field $\mathbf{F}$. They are surrounded by four ring electrodes (yellow, two of them are not represented). At the center $O$ of the structure, the atoms interact with three laser beams (780 and $776 \mathrm{~nm}, \sigma^{+}$polarized, red arrow; $1258 \mathrm{~nm}, \pi$ polarized, green arrow) that cross at $90^{\circ}$ in the horizontal plane. At the end of the sequence, the atoms are detected in the state-selective fieldionization detector $D$. 
interacted with the rf field, the atoms drift toward another capacitor, where they are detected by state-selective field ionization. The detector resolves levels of different manifolds or with very different $m$ values in the same manifold. We measure the population of levels with overlapping fieldionization signals by applying additional probe microwave (MW) pulses, as described in Appendix C.

\section{FAST CIRCULARIZATION}

The standard procedure for rf-induced circular state preparation is a rapid adiabatic passage sequence [33], in which the electric field is slowly ramped down across $F_{0}$ in the presence of the rf field. Since the transition frequency $\omega_{2,3}$ between $52 m_{2}$ and $52 m_{3}$ only differs from $\omega_{n}$ by a few megahertz for $F \approx F_{0}$, scanning the field over $0.24 \mathrm{~V} / \mathrm{cm}$ transfers the atoms from $52 m_{2}$ into $52 c$ with a $99.5 \%$ efficiency, cf. Appendix D. To reach such a high efficiency, the adiabatic passage method has to be slow, with a preparation time in the few microsecond range at least. Such a long time can be detrimental, in particular for quantum sensing experiments [4,34].

The $52 c$ state can be prepared much faster from the same $52 m_{2}$ level by setting $F=F_{0}$ and applying a constant amplitude rf pulse [33]. The duration of the preparation (96 ns) is only limited by the applied rf amplitude. Figure 3 shows the time evolution of the populations of relevant states. The maximum transfer efficiency is limited to 77.4 (4)\%, significantly lower than that of the adiabatic passage. Some of the population remains trapped in noncircular levels, due to the frequency shifts of the $m=0$ and $m=1$ levels induced by the quantum defects.

Optimal control allows us to combine fast preparation with high fidelity. We use as experimental knobs two timedependent voltages, $V_{r}(t)$ and $V_{i}(t)$, generated by two

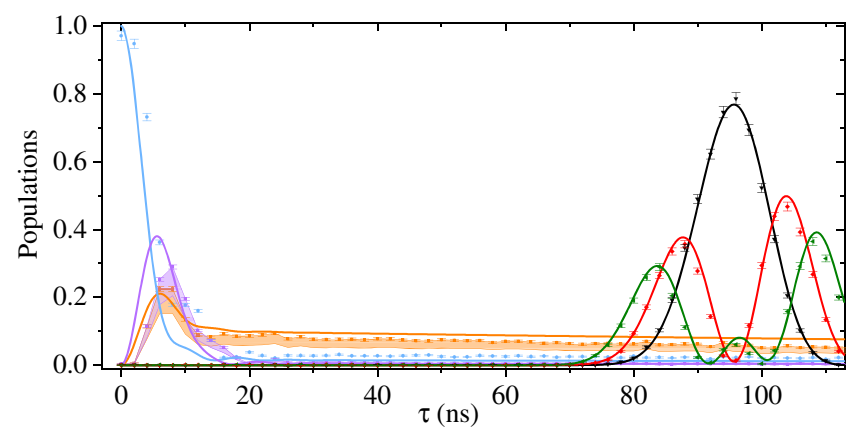

FIG. 3. Circular state preparation with a square pulse. Evolution of the population $P_{j}(\tau)$ of the levels labeled by $j\left(j=m_{1}\right.$, $m_{2}, m_{3}, e_{2}, e_{1}$, and $c$, where the labels and the associated color code are defined in Fig. 1) as a function of the duration $\tau$ of the rf pulse. The points are experimental with statistical error bars. The shaded areas (purple and orange) shows the error range for levels $m_{1}$ and $m_{3}$ due to the uncertainty on the MW probe pulses calibration (see Appendix C). The solid lines correspond to the numerical simulation of the experiment. outputs of an arbitrary waveform generator (Appendix E). They control rf mixers which modulate the amplitudes $F_{r}(t)$ and $F_{i}(t)$ of the two quadratures of the $\sigma^{+}$-polarized rf field:

$$
\begin{aligned}
\mathbf{F}_{\mathrm{rf}}(t)= & F_{r}(t)\left[\cos \left(\omega_{\mathrm{rf}} t\right) \mathbf{u}_{x}+\sin \left(\omega_{\mathrm{rf}} t\right) \mathbf{u}_{y}\right] \\
& +F_{i}(t)\left[-\sin \left(\omega_{\mathrm{rf}} t\right) \mathbf{u}_{x}+\cos \left(\omega_{\mathrm{rf}} t\right) \mathbf{u}_{y}\right] .
\end{aligned}
$$

The process we seek to optimize is a state-to-state transfer from the initial state, $52 m_{2}$, to the target state $\left|\Psi_{\text {tgt }}\right\rangle, 52 c$ [35]. The success of the transfer is quantified by the realvalued target functional,

$$
J_{T}=1-\left|\left\langle\Psi(T) \mid \Psi_{\mathrm{tgt}}\right\rangle\right|^{2},
$$

where $|\Psi(T)\rangle$ is the final state at the end of the protocol. A perfect match of final and target state, up to a global phase, is obtained if and only if $J_{T}$ takes its minimal value, $J_{T}=0$. The final state depends implicitly, via its time evolution, on the external controls $F_{r}(t)$ and $F_{i}(t)$. An optimal choice of $F_{r / i}(t)$ can be calculated by minimization of the target functional [8]. Here, we use Krotov's method to minimize $J_{T}$. It is a gradient-based optimization technique that ensures monotonic convergence of $J_{T}$ toward its minimum [38]. A detailed, hands-on introduction of the method is found in Ref. [39]. As with any gradient-based technique, the condition for $J_{T}$ to be extremal, $\nabla J=0$, results in an update equation for the external controls, and evaluation of the gradient implies forward propagation in time of the initial state and backward propagation of the target state [8]. In practice, the functional is thus minimized iteratively by updating $F_{r}(t)$ and $F_{i}(t)$ until we reach a transfer efficiency of $99 \%$ in the simulation.

In our system, the rf pulse mainly couples $m$ levels on the lowest diagonal in Fig. 1, but off-resonant excitation may also populate the levels on the second lowest diagonal. These levels are thus included in the calculation. To ensure that the optimized field is experimentally feasible, we need to include constraints on the spectral bandwidth and the maximum amplitude of the pulse. The simplest possible implementation-truncation of the optimized pulse to the allowed range of amplitude and frequency after each iteration step-has turned out to be sufficient to this end [35].

The optimized pulse is presented Fig. 4(a). The dynamics induced by the shaped pulse is discussed in detail in Ref. [35]. Briefly, the optimized pulse can be decomposed into two phases [separated by a vertical line in Fig. 4(c)]. The first $40 \mathrm{~ns}$ prepare a superposition of hydrogenic levels $(m>2)$ that corresponds to a spin coherent state (SCS) on the Bloch sphere, while leaving no spurious population in $m=1$ [Figs. 4(c) and 4(d)]. The last 73 ns rotate the SCS onto the circular state.

The optimization algorithm produces a pulse, in which the amplitude of the two quadratures is modulated even 

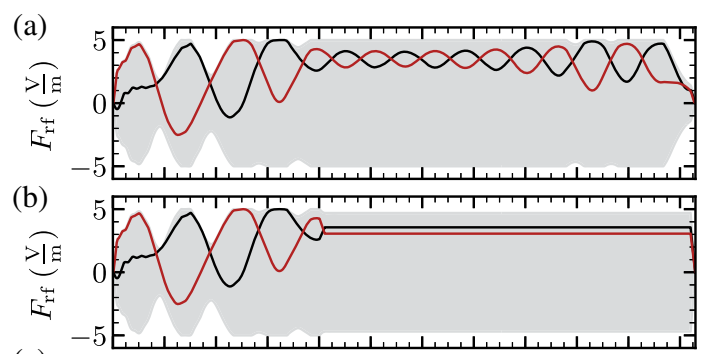

(c)

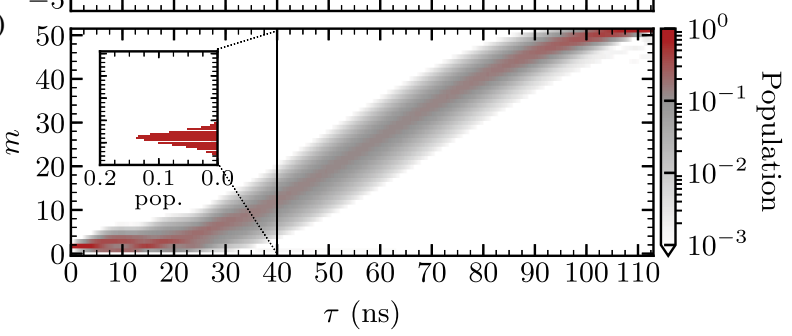

(d)

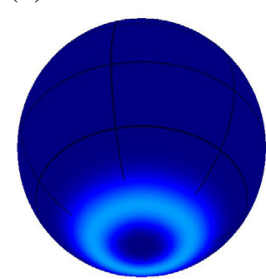

(i)

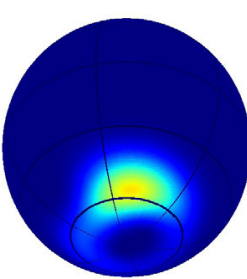

(ii)

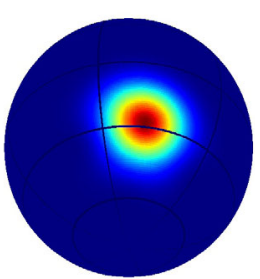

(iii)
FIG. 4. Optimized circularization pulse. Amplitude of the two quadratures (red and black lines) of the optimized rf pulse (a) before and (b) after postprocessing (flattening of the oscillations that result from the constraint on the spectral bandwidth of the pulse). The gray area indicates the pulse envelope. (c) Evolution of the population of each Stark level of the lower diagonal as a function of time for the rf pulse of (b). The inset presents the histogram of the populations at $\tau=40 \mathrm{~ns}$ (red bars). (d) Snapshot of the $Q$ function of the atomic state represented as a large angular momentum on a generalized Bloch sphere for $\tau=0 \mathrm{~ns}$ (i), $\tau=15 \mathrm{~ns}$ (ii), and $\tau=40 \mathrm{~ns}$ (iii). The first $40 \mathrm{~ns}$ of the pulse transfer the $52 m_{2}$ level (i) into a spin coherent state (iii).

after $\tau>40 \mathrm{~ns}$. This corresponds to a slow oscillation of the direction of the rotation axis of $\mathbf{J}$ on the Bloch sphere. This modulation is not necessary for reaching the circular state. It results from the constraint on the spectral bandwidth that is imposed during the optimization. The physical insight in the dynamics under the optimized pulse allows us to remove the unnecessary modulation. We have checked numerically that, after $t>40 \mathrm{~ns}$, these oscillations can be flattened out into a pulse of constant amplitude and wellchosen phase without losing the transfer efficiency into the circular state [Figs. 4(b) and 4(c)].

Using the rf mixer calibration (Appendix E), we calculate, from the flattened theoretical pulse, the voltage $V_{r}(t)$ and $V_{i}(t)$ to be applied in the experiment. We finally perform a "closed-loop" optimization, directly on the experimental signal. For the first $40 \mathrm{~ns}$ the two quadratures

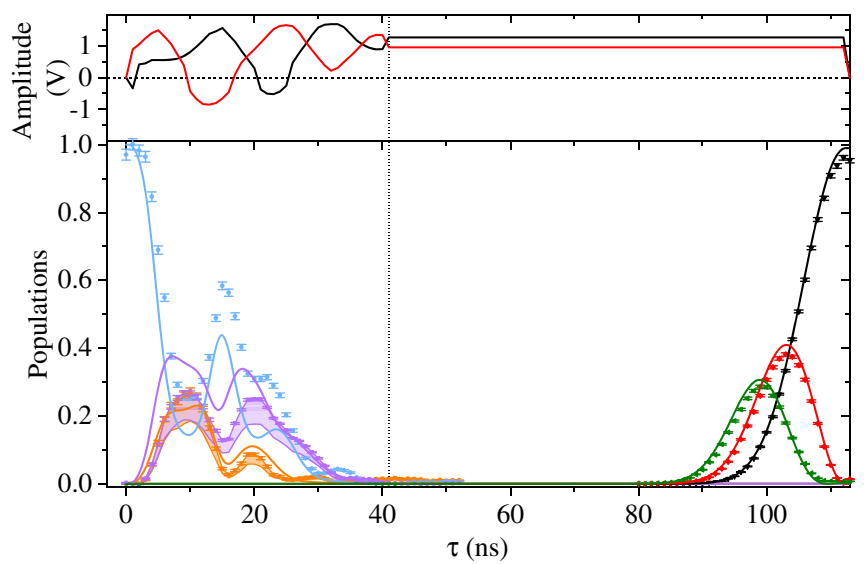

FIG. 5. Circular state preparation with an optimized pulse. The voltage $V_{r}$ and $V_{i}$ for the optimized pulse are shown in the upper panel. The main frame shows the evolution of the population $P_{j}(\tau)$ of the levels $j$ as a function of the duration $\tau$, after which the rf pulse is interrupted, for $j=m_{1}, m_{2}, m_{3}$ ( $\left.\tau \leq 52 \mathrm{~ns}\right)$ and $j=$ $e_{2}, e_{1}, c(\tau \geq 80 \mathrm{~ns})$. The points are experimental with statistical error bars, using the color code of Fig. 1. The shaded area (purple and orange) shows the error range for levels $m_{1}$ and $m_{3}$ due to the uncertainty on the MW probe pulses calibration. The solid lines correspond to the numerical simulation of the experiment. The discrepancy between the data and the simulation at the beginning of the optimized pulse is probably due to the finite bandwidth of the rf circuit. When we set $V_{r}$ and $V_{i}$ to zero at time $\tau$, the rf has a finite ring-down time, which affects the measured population. The effect of this ring down is not visible at the end of the pulse, as it corresponds there to a small additional rotation of the spin, which is automatically compensated by the optimization procedure. Finally, we find $P_{c}=96.2(3) \%, P_{e_{1}}=0.20(1) \%, P_{e_{2}}=0.74(4) \%$. We also measure $P_{e_{3}}=0.15(7) \%, P_{e_{4}}=0.13(8) \%, P_{m_{3}}=0.08(3) \%$, $P_{m_{2}}=0.37(4) \%, P_{m_{1}}=1.16(8) \%$.

are rescaled by the same 0.95 factor, close to 1 , in order to compensate for the rf amplitude calibration uncertainty. For the flat part of the pulse ( $\tau>40 \mathrm{~ns})$, we finely tune the SCS rotation by independently adjusting the constant values of $V_{r}(t)$ and $V_{i}(t)$ in order to optimize the final circular state population (Appendix F).

Figure 5 presents the experimental evolution of the Stark levels populations (dots) for the optimized pulse (abruptly interrupted after a variable duration $\tau$ ). The transfer probability into the circular state now reaches $96.2(3) \%$, for a preparation time (113 ns) comparable to the duration of the square pulse (Fig. 3). The fact that only about 25 oscillations of the carrier rf wave perform such an efficient transfer is a remarkable result. The evolutions of the probed level populations are in good agreement with the numerical predictions (solid lines). Quantum control provides thus a considerable improvement over the usual circular state preparation methods.

We now test the coherence of the prepared state by checking that the process does not spoil a coherent superposition with a reference level. For practical reasons, we 

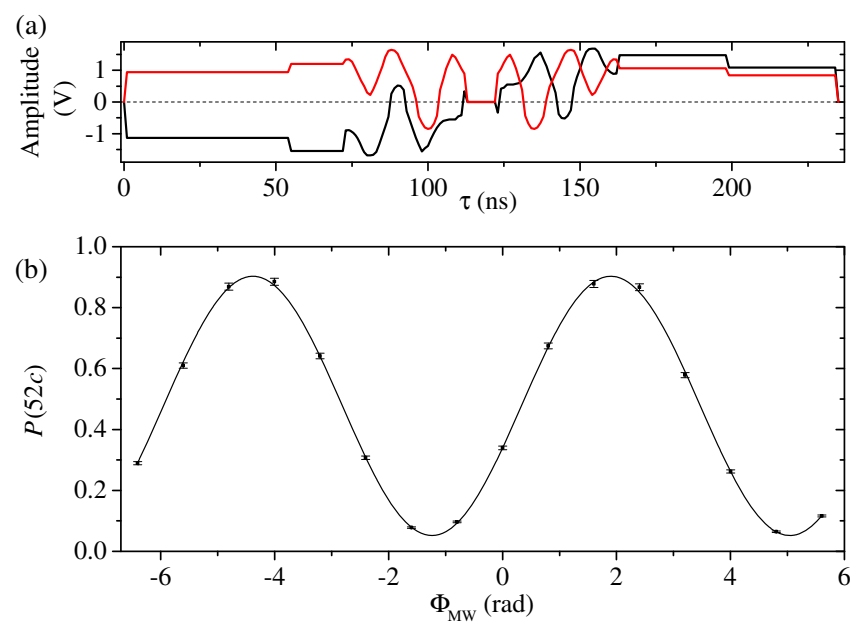

FIG. 6. Coherence test. (a) Evolution of the control voltages $V_{r}(\tau)$ (black line) and $V_{i}(\tau)$ (red line) in the sequence that tests the coherence of the optimized circularization pulse. We first apply a time-reversed optimized pulse followed by a circularization pulse similar to that of Fig. 2(b). The flat parts of the pulses $(0 \leq \tau \leq 73$ and $163 \leq \tau \leq 236 \mathrm{~ns})$ are now divided in two steps, ensuring that the reference $50 \mathrm{c}$ level is transferred back into itself by each pulse. (b) Probability to finally detect the atoms in $52 c$ as a function of the relative phase between the MW Ramsey pulses. The points are experimental with statistical error bars, the solid line is a sine fit. The visibility [89.1(6)\%] is excellent, limited mostly by the technical electric field noise in the experiment.

start from a superposition of $52 c$ (reached by an initial rapid adiabatic passage) and the reference level 50c. This superposition is prepared by a $\pi / 2 \mathrm{mw}$ pulse driving the $52 \mathrm{c} \rightarrow$ $50 c$ two-photon transition. A sequence of a time-reversed optimized rf pulse (Appendix F) and of a direct one, separated by a $10 \mathrm{~ns}$ delay, drives $52 c$ into $52 m_{2}$ and back. A final $\pi / 2 \mathrm{mw}$ pulse mixes again $52 c$ and $50 c$ and closes a Ramsey interferometer.

The optimized time-reversed and direct pulses are shown in Fig. 6(a). Since the Stark frequency $\omega_{50} / 2 \pi$ differs by only $10 \mathrm{MHz}$ from $\omega_{r f} / 2 \pi$, the rf driving produces a spurious rotation of the spin associated to the $n=50$ manifold. By using two amplitude levels for the first $73 \mathrm{~ns}$ of the reversed pulse and the last $73 \mathrm{~ns}$ of the direct pulse, we ensure that each pulse returns $50 c$ exactly onto itself, while preserving the optimal transfer between $52 c$ and $52 m_{2}$ (Appendix F). Figure 6(b) presents the probability for finally detecting the atoms in $52 c$ as a function of the relative phase of the two MW pulses. The fringe visibility is 0.891(6), a large value, limited by electric field noise. The atoms are transiently cast in a superposition of levels $\left(52 m_{2}\right.$ and $50 c$ ) with very different dipoles which is utterly sensitive to the electric field [4]. An extrapolation to zero electric noise leads to a visibility equal to 1 within the error bars (Appendix G). We thus conclude that the rf optimized pulse preserves coherences extremely well.

\section{CAT STATE PREPARATION}

Optimal control opens much wider possibilities than merely improving circular state preparation. We use the same methodology as described above, simply replacing the target state $\left|\Psi_{\text {tgt }}\right\rangle$ by an equal weight superposition of the lowest level in the $m=1$ manifold $\left(52 m_{1}\right)$ with the circular state $(52 c)$. This superposition is a Schrödingercat-like state useful for quantum-enabled electrometry [4]. It is particularly challenging to prepare, as its preparation is equivalent to a $\pi / 2$ pulse on a 50 -photon transition.

We again design the rf pulse driving the desired state transfer using Krotov's optimization method. Figure 7(a) presents the corresponding results, with Figs. 7(b) and 7(c) showing the calculated evolution of the population in both the $m$ level basis and the dressed-state basis. The preparation of the cat state involves a transient superposition of many of the dressed states in a complex interference process. During the first tens of nanoseconds, the optimized pulse progressively shelves half of the population into the eigenstate of the atom-rf field Hamiltonian that has the largest $52 m_{1}$ component [nearly horizontal line in Fig. 7(c)] while bringing the other half of the wave function into a SCS [inset of Fig. 7(b)]. The second part of the pulse rotates the SCS toward the north pole of the Bloch sphere, while leaving the population of $52 m_{1}$ unaffected (the eigenstate is a dark state for the rf). At the end of the pulse, as the SCS

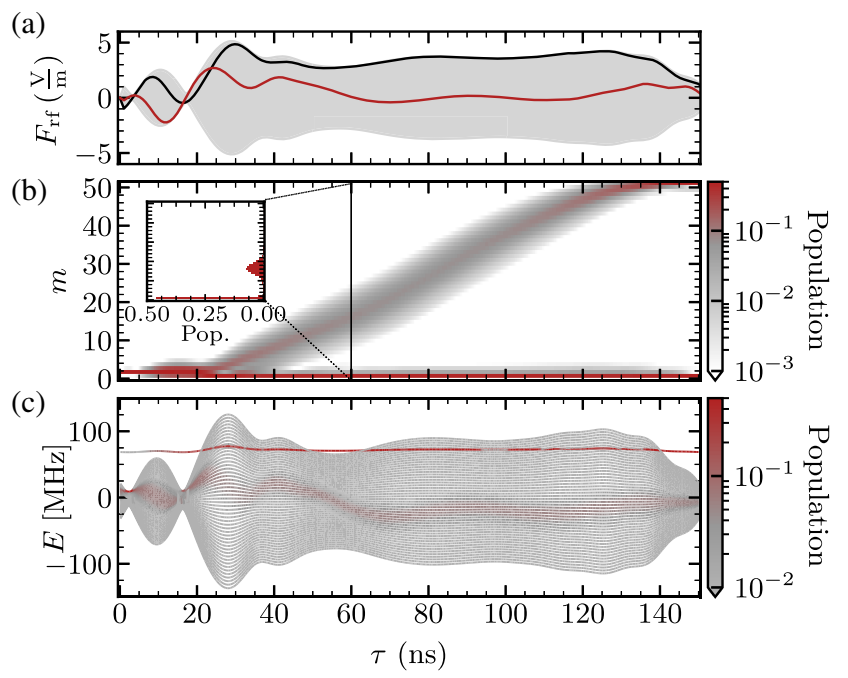

FIG. 7. Optimized cat state preparation pulse. (a) Amplitude of the two quadratures of the rf pulse given by the Krotov optimization. Time evolution of the population of each Stark level of the lower diagonal (b) and of the instantaneous eigenstates of the atom-rf field Hamiltonian in the rotating frame (c), denoted as "dressed states" in the main text. The vertical position of the lines in (c) reflects the instantaneous energy of the corresponding state, and the color of a line shows its population at time $\tau$. The inset in (b) presents the computed population histogram for the different $m$ levels at $\tau=60 \mathrm{~ns}$, showing that the pulse prepares, during the first few tens of nanoseconds, a superposition of $m=1$ and a spin coherent state made up of levels with $m>2$. 


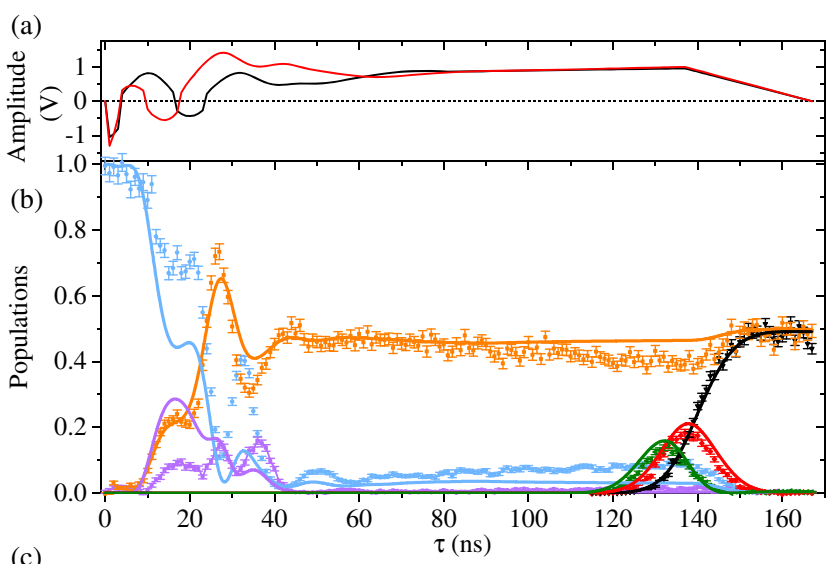

(c)

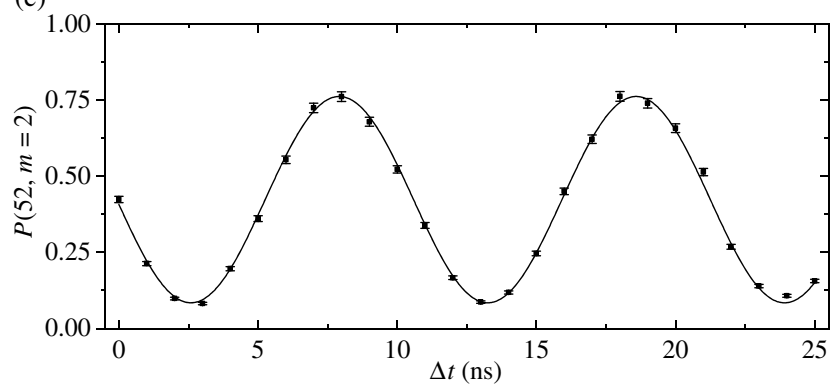

FIG. 8. Cat state preparation with the optimized pulse. (a) Control voltages $V_{r}(\tau)$ (black line) and $V_{i}(\tau)$ (red line), applied to produce the superposition of $52 m_{1}$ and $52 c$, as a function of time. (b) Time evolution of the population $P_{j}(\tau)$ of level $j\left(j=m_{1}, m_{2}\right.$, $m_{3}, e_{2}, e_{1}$, and $c$ ). The points are experimental with statistical error bars, using the color code of Fig. 1. The solid lines correspond to the theoretical prediction for the optimized pulse. The final target state population (averaged over the last six points) is $P_{c}=48(1) \%$ and $P_{m_{1}}=48.4(8) \%$. (c) Probability to find the atoms in the $52 m_{2}$ state after the pulse (a) followed by the time-reversed optimized pulse that recombines $52 m_{1}$ and $52 c$ (Appendix $\mathrm{F}$ ) as a function of the delay $\Delta t$ between the pulses. The points are experimental with statistical error bars, the solid line is a sine fit. The large fringe visibility, mostly limited by the technical electric field noise, demonstrates the coherence of the cat state prepared by the first pulse.

reaches the circular state, the rf is slowly switched off so that the part of the wave function in the dark state adiabatically transfers into a pure $52 m_{1}$ level.

Similarly to the case of circular state preparation, the qualitative understanding of the dynamics allows us to simplify the pulse. We replace the pulse in the second half of the protocol ( $\tau>80 \mathrm{~ns})$ by a linearly increasing and decreasing amplitude of the control voltage of each quadrature (Appendix F). This enables us to tune the rotation angle of the spin coherent state (by independently changing the pulse area for each quadrature), while ensuring that the phase and amplitude of the rf field vary slowly enough for the other half of the wave function to adiabatically remain shelved in the instantaneous dressed state.

Figure 8(a) shows the programmed pulse. Its shape includes minor compensations for the finite electronic bandwidth, and the parameters of the two linear amplitude ramps are adjusted to optimize the rotation of the SCS (Appendix F). Figure 8(b) shows the experimental evolution of the relevant level populations (dots). The preparation of the dressed state with a large component of $52 m_{1}$ is evidenced by the nearly constant population of $52 m_{1}$ after $60 \mathrm{~ns}$. The final population balance between $52 m_{1}$ and $52 \mathrm{c}$ is excellent. The experiment is again in good agreement with the numerical model (solid lines).

In order to test the coherence of the cat state, we apply, after an adjustable delay $\Delta t$, a time-reversed preparation pulse. During the delay, the superposition state accumulates a phase in the frame rotating with the rf carrier frequency. The state at the end of the time-reversed pulse thus oscillates between $52 m_{2}$ and a state orthogonal to it as a function of $\Delta t$. Figure 8(c) shows the oscillations, with $\Delta t$, of the probability to find the atoms finally in $52 m_{2}$. The visibility of the interference is high [0.80(1)]. An extrapolation to zero electric field noise (Appendix G) leads to a visibility of $0.97(2)$. We estimate that we prepare the expected state superposition with a remarkable $93 \%$ fidelity.

\section{CONCLUSIONS}

We have used quantum optimal control to prepare nontrivial Rydberg states with high fidelity in short times. The optimized pulses are very robust to the limited calibration of the control electronics. At the same time, they are comprehensible, which eases adaptation of the pulses to the experimental constraints. For example, the strategy for the cat state pulse consists in preparing a superposition of a spin coherent state and an eigenstate that is kept dark during the rotation of the spin coherent state. This understanding allows us to experimentally fine-tune the final rotation of the spin coherent state without affecting the final population of $52 m_{1}$.

The preparation could be made even faster if more $\mathrm{rf}$ power is available in the experiment. The method could be extended to a larger variety of Rydberg quantum states, in particular by applying simultaneously $\sigma^{+}$- and $\sigma^{-}$-polarized shaped pulses, with interesting applications for quantum-enabled electrometry and magnetometry $[4,34]$. More generally, our results attest for the power and reliability of quantum optimal control in a Hilbert space of a large dimension, with a complex combination of harmonic and anharmonic ladders. They open the way for quantum optimal control based state engineering in complex systems for applications in quantum science and technology.

\section{ACKNOWLEDGMENTS}

Financial support from the Agence Nationale de la Recherche under the project "SNOCAR" (167754) and the Studienstiftung des deutschen Volkes is gratefully acknowledged. This publication has received funding from the European Union's Horizon 2020 research and 
innovation programme under Grant Agreement No. 817482 (PASQuanS).

\section{APPENDIX A: NUMERICAL OPTIMIZATION}

In this appendix, we describe additional details of the pulse optimization (see also Ref. [35] for a comprehensive description).

In order to calculate the dynamics of the atoms, we first numerically compute all the eigenvalues of the Stark Hamiltonian (in the absence of rf field) for each magnetic quantum number $m \geq 0$. For this diagonalization we consider all states with a principal quantum number of $n=52 \pm \Delta n$. We use the known rubidium quantum defects up to $l=7$ [40] for describing the energy levels in zero electric field. We then compute the transition matrix elements between neighboring $m$ and $m+1$ Stark levels. We check the validity of the Hilbert space truncation by comparing the eigenstates and transition matrix elements for one value of $\Delta n$ with an extended Hilbert space with $\Delta n+1$. For the chosen value of $\Delta n=4$, this method leads to an accuracy of the order of $10^{-6}$ in the energy levels and transition matrix elements.

At the field strength $F=F_{0}=2.5 \mathrm{~V} / \mathrm{cm}$, we find transition frequencies $\omega_{m, m+1}$ between $m$ and $m+1$ :

$$
\begin{aligned}
& \omega_{0,1}=2 \pi \times 71.1 \mathrm{MHz}, \\
& \omega_{1,2}=2 \pi \times 190.3 \mathrm{MHz}, \\
& \omega_{2,3}=2 \pi \times 244.8 \mathrm{MHz} .
\end{aligned}
$$

For $m \geq 3$, all the transition frequencies $\omega_{m, m+1} / 2 \pi$ are less than $1 \mathrm{MHz}$ away from $\omega_{\mathrm{rf}} / 2 \pi$ (the difference being due to residual quantum defect shifts and the second-order Stark effect).

\section{Fast circularization}

The optimization of the desired state-to-state transfer in the Stark manifold of the rubidium Rydberg atom was performed using Krotov's method [35]. We have assumed the initial state to be the lower state of the $52 m=2$ ladder of the Stark manifold. For this optimization, it is sufficient to take the lowest two diagonal ladders of the $n=52$ manifold into account and to operate thus in a Hilbert space of dimension 103 [35]. As a final check, we perform a numerical integration with the full, untruncated Hamiltonian using the optimized pulse. We find the same final population of the target state as in the truncated space within a precision of $5 \times 10^{-5}$.

In the optimization, we take into account two experimental limitations. At each iteration of the optimization, we truncate the pulse bandwidth by a square window (frequency bounds $140 \leq \omega_{\mathrm{rf}} / 2 \pi \leq 360 \mathrm{MHz}$, with sineshaped edges of $20 \mathrm{MHz}$ width). This avoids getting variations of the quadrature amplitudes that would be too fast to be implemented experimentally. We also limit the pulse amplitude by truncating the pulse to the maximal experimentally available value $F_{\text {rf }}=5 \mathrm{~V} / \mathrm{m}$.

We then carry out the optimization for a given total pulse duration, targeting a preparation fidelity larger than $99 \%$. The guess pulse has a flattop shape with sine-shaped edges with a rise and fall time of 10 ns. For too short pulse durations, the algorithm does not converge within the imposed limitations; it first reaches convergence for a duration of $113 \mathrm{~ns}$.

\section{Cat state preparation}

The target state for this optimization is chosen to be $\left(\left|52 m_{1}\right\rangle-|52 \mathrm{c}\rangle\right) / \sqrt{2}$. The maximal rf amplitude was kept at $F_{\text {rf }}=5 \mathrm{~V} / \mathrm{m}$, but the bandwidth of the pulse was slightly changed to $130 \leq \omega_{\mathrm{rf}} / 2 \pi \leq 320 \mathrm{MHz}$ with sineshaped edges of $50 \mathrm{MHz}$ width, such that the center of the frequency window is a bit lower than for the circularization pulse. The guess pulse was similar to the circularization one. The duration of the pulse had to be increased to $150 \mathrm{~ns}$ for a successful optimization.

\section{APPENDIX B: TIMING OF THE EXPERIMENT}

The setup is depicted Fig. 2. A thermal beam of Rb, effusing from an oven, crosses a structure made of two planeparallel electrodes surrounded by four electrodes that form a ring around them. At the center of the structure, the atoms interact with three laser beams at 780, 776, and $1258 \mathrm{~nm}$, resonant with the $5 S_{1 / 2} \rightarrow 5 P_{3 / 2}, 5 P_{3 / 2} \rightarrow 5 D_{5 / 2}$, and $5 D_{5 / 2} \rightarrow 52 F$ transitions, respectively. The 780 and $776 \mathrm{~nm}$ propagate along the same direction, and are always on. The $1258 \mathrm{~nm}$ laser is sent perpendicular to the other laser beams, and is switched on for $1 \mu \mathrm{s}$ at the beginning of the sequence. The beginning of the laser pulse sets the time origin $t=0$.

During the laser excitation, the quantization axis is defined by a small horizontal electric field along the axis of the 780 and $776 \mathrm{~nm}$ lasers, created by a voltage applied across two of the ring electrodes. This field partially lifts the degeneracy of the $52 \mathrm{~F}$ level, and we set the $1258 \mathrm{~nm}$ laser to be on resonance with the transition toward the $52 \mathrm{~F}$ sublevel with $|m|=2$. We also choose the polarization of the 780 and $776 \mathrm{~nm}$ lasers to be $\sigma^{+}$polarized and the $1258 \mathrm{~nm}$ laser to be $\pi$ polarized with respect to the quantization axis. We thus prepare the atoms selectively in the state $|52 F, m=2\rangle$.

Once the atoms have been excited to the Rydberg state, the electric field is adiabatically rotated and ramped up to $F=2.63 \mathrm{~V} / \mathrm{cm}$ between $t=2 \mu \mathrm{s}$ and $t=3 \mu \mathrm{s}$. It is finally aligned with $O z$, which is the quantization axis for the rest of the experiment. It remains constant for $1.3 \mu \mathrm{s}$, and decreases down to $F=2.39 \mathrm{~V} / \mathrm{m}$ in $1.5 \mu \mathrm{s}$. It is finally kept constant for $1 \mu \mathrm{s}$. This variation of the electric field makes it possible to prepare the $52 c$ circular state with 
a high purity by adiabatic passage if needed as initial state for the rest of the sequence. In this case, we ramp up at $t=2.91 \mu$ s the amplitude of the rf field to a value of $4.8 \mathrm{~V} / \mathrm{m}$ in $2.05 \mu \mathrm{s}$. We leave the amplitude constant for $0.1 \mu \mathrm{s}$, and ramp it down to zero in $2.05 \mu \mathrm{s}$. When decreasing $F=2.63 \mathrm{~V} / \mathrm{cm}$ down to $F=2.39 \mathrm{~V} / \mathrm{cm}$, the Stark frequency in $n=52$ varies between 262 and $238 \mathrm{MHz}$ and crosses the rf source frequency while the rf amplitude is near its maximum. Ramping down the electric field in the presence of the rf adiabatically transfers the atoms from $52 m_{2}$ into the circular state. If we choose not to apply the rf, the atoms remain in $52 m_{2}$. At $t=7 \mu \mathrm{s}$, we set the amplitude of the electric field to the value $F_{0}$, and we start the optimized rf pulse at $t=8.71 \mu \mathrm{s}$.

\section{APPENDIX C: MEASUREMENT OF LEVEL POPULATIONS}

The field-ionization detection method does not resolve Stark sublevels of the same manifold that have similar $m$ values. Therefore, to measure the population of a given sublevel of the $n=52$ manifold after the rf pulse, we selectively transfer its population into a level inside the 51 or 50 manifolds with a MW $\pi$ pulse, called "probe pulse," and count the number of atoms in the target manifold. However, different atomic levels may have different detection efficiencies (Fig. 10). As a result, we use specific normalization methods for each relevant level.

\section{Low-m states}

We measure the population of the levels $52 m_{i}(i=1,2$, 3 ) by applying, after the end of the rf pulse, a MW probe $\pi$ pulse tuned on one of the $52 m_{i} \rightarrow 51 m_{2}$ transitions. In order to avoid a strong count rate reduction due to the finite lifetime of $51 \mathrm{~m}_{2}$, we then transfer the population of $51 \mathrm{~m}_{2}$ to $51 \mathrm{c}$ by adiabatic passage and count the number of atoms in the $51 c$ state. This number is proportional to the population of the probed levels and to the efficiencies of the $52 m_{i} \rightarrow$ $51 m_{2} \pi$ pulses. They are carefully calibrated and included in the calculation of populations in $52 m_{i}$. These populations are obtained by using as a normalization factor the number of atoms counted in $52 m_{2}$ (via 51c) in the absence of $\mathrm{rf}$ pulse. Note that this method discriminates the states with $m=2$ and with $m=-2$. Atoms initially prepared in the $52, m=-2$ state due to small laser polarization imperfection are transferred in $51, m=-2$ by the probe MW $\pi$ pulse but are not transferred into $51 c$ by the $\sigma^{+}$rf circularization pulse.

The $52 m_{i} \rightarrow 51 m_{2}(i=1,3)$ transitions have a large linear Stark effect. A slow drift of the static electric field $\left(\sim 10^{-4} \mathrm{~V} / \mathrm{m}\right.$ over an hour timescale) results in significant errors in the calibration of MW $\pi$ pulse efficiencies (shaded area on Fig. 3 of the main text). For the cat state preparation (Fig. 8 of the main text), we normalize the population in $52 m_{1}$ by the number of atoms directly prepared in $52 m_{1}$

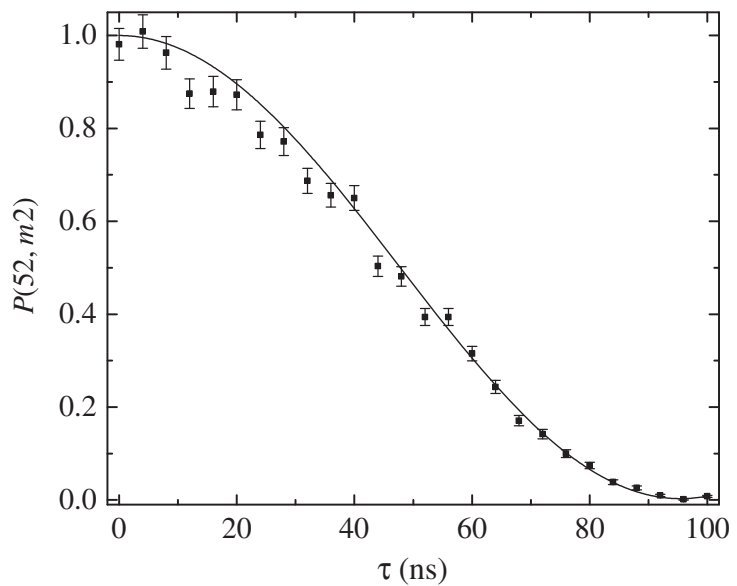

FIG. 9. Preparation of the $52 m_{1}$ state. To normalize the population of $52 m_{1}$ in the superposition state, we modulate the two quadratures of the $250 \mathrm{MHz}$ carrier at $59.5 \mathrm{MHz}$ in order to generate a $190.5 \mathrm{MHz}$ rf field that is resonant with the $52 m_{2}-$ $52 m_{1}$ transition. The points represent the population $P_{m_{2}}$ remaining in the $52 m_{2}$ state as a function of duration $\tau$ of the pulse. For $\tau=88 \mathrm{~ns}$, we measure $P_{m_{2}} \approx 0.3 \%$. The amplitude of the rf is chosen low enough so that the atoms cannot be transferred into $52 m_{3}$ (numerical simulations estimate that $P_{m_{3}}<0.5 \%$ ). The pulse thus prepares $51 m_{1}$ with more than $99 \%$ efficiency.

from $52 m_{2}$ using a resonant $\mathrm{rf} \pi$ pulse (efficiency $99 \%$ as seen on Fig. 9). This method is insensitive to the $52 m_{1} \rightarrow$ $51 m_{2}$ MW probe pulse efficiency calibration.

\section{High-m states}

We measure the population of the $52 c, 52 e_{i}(i=1,2,3$, 4 ), and $52 e_{1}^{\prime}$ levels, by applying a probe microwave $\pi$ pulse that selectively transfers one of these levels into the corresponding one in the 50 manifold [33], which is finally detected. For each of these levels, we calibrate a correction factor that takes into account the efficiency of the microwave $\pi$ pulses and the relative detection efficiency of relevant levels in order to estimate their populations [41]. We normalize populations by the number of atoms that we detect with the $52 c$ probe when we prepare the circular state by adiabatic passage. This method thus directly compares the efficiency of the preparation of the circular state using the optimized pulse with that of the adiabatic passage (estimated at $\sim 99.5 \%$; see below), independently from the transfer efficiency of the circular state MW probe.

\section{APPENDIX D: ADIABATIC PASSAGE EFFICIENCY}

Figure 10 presents the ionization signals of the atomic state with and without performing the adiabatic passage at $t=2.91 \mu \mathrm{s}$. We clearly see that most of the population in the initial state has been transferred to high- $m$ states, which ionize in larger fields. The residual counts at the position of the $52 m_{2}$ ionization peak (about $0.5 \%$ ) most likely 


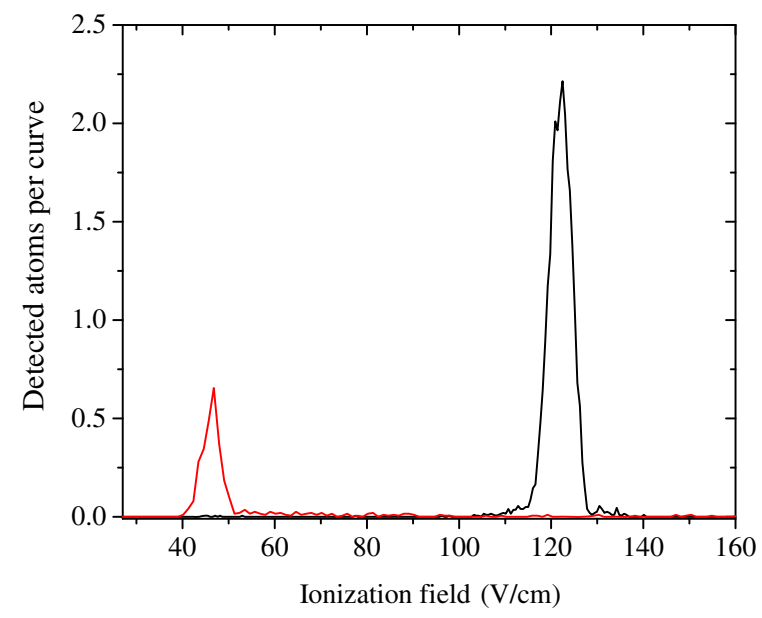

FIG. 10. Ionization signals. Number of detected atoms per sequence as function of the ionization field applied in the fieldionization detector for the laser-excited $52 m_{2}$ state (red line) and for the $52 c$ state prepared by the adiabatic passage (black line). The difference of height of the two peaks results from the shorter lifetime of the $52 m_{2}$ state. The residual peak in the black signal at the position of the red one probably results from Rydberg atoms spuriously prepared in the state $m=-2$ due to laser polarization imperfections.

correspond to atoms that have been prepared in the $m=-2$ state (due to the excitation laser polarization imperfections) and do not interact with the $\sigma^{+}$radio frequency. However, our detection method using probes distinguishes between $m= \pm 2$ (see above). The $m=-2$ atoms thus do not contribute to the measured populations in the $m=2$ state.

There are two possible effects that limit the efficiency of the adiabatic passage [33]. On the one hand, if the adiabaticity criterium is not fulfilled, some population can remain in the second last level of the energy ladder, $52 e_{1}$. On the other hand, if the polarization of the radio frequency is not purely $\sigma^{+}$, some population can end up in the elliptical state $52 e_{1}^{\prime}$, defined on Fig. 1 . In order to precisely quantify the purity of the adiabatic circular state preparation, we have measured the population of the $52 e_{1}$ and $52 e_{1}^{\prime}$ states after the adiabatic passage using probes [33]. We find $P_{e_{1}}=0.15(1) \%$ and $P_{e_{1}^{\prime}}=0.39(3) \%$, respectively. Since these numbers are already very low, the probability for the atoms to be in levels further away from the circular is negligible. We thus estimate a preparation fidelity on the order of $99.5 \%$.

\section{APPENDIX E: rf SETUP}

We generate the two quadratures of the radio-frequency fields by using two sets of four synthesizers with a global phase difference of $\pi / 2$. For each set, we optimize the relative phases and amplitudes in order to generate a purely $\sigma^{+}$-polarized rf field with the four ring electrodes. We control the field global amplitude of each quadrature by applying control voltages $V_{r}(t)$ and $V_{i}(t)$ to sets of mixers

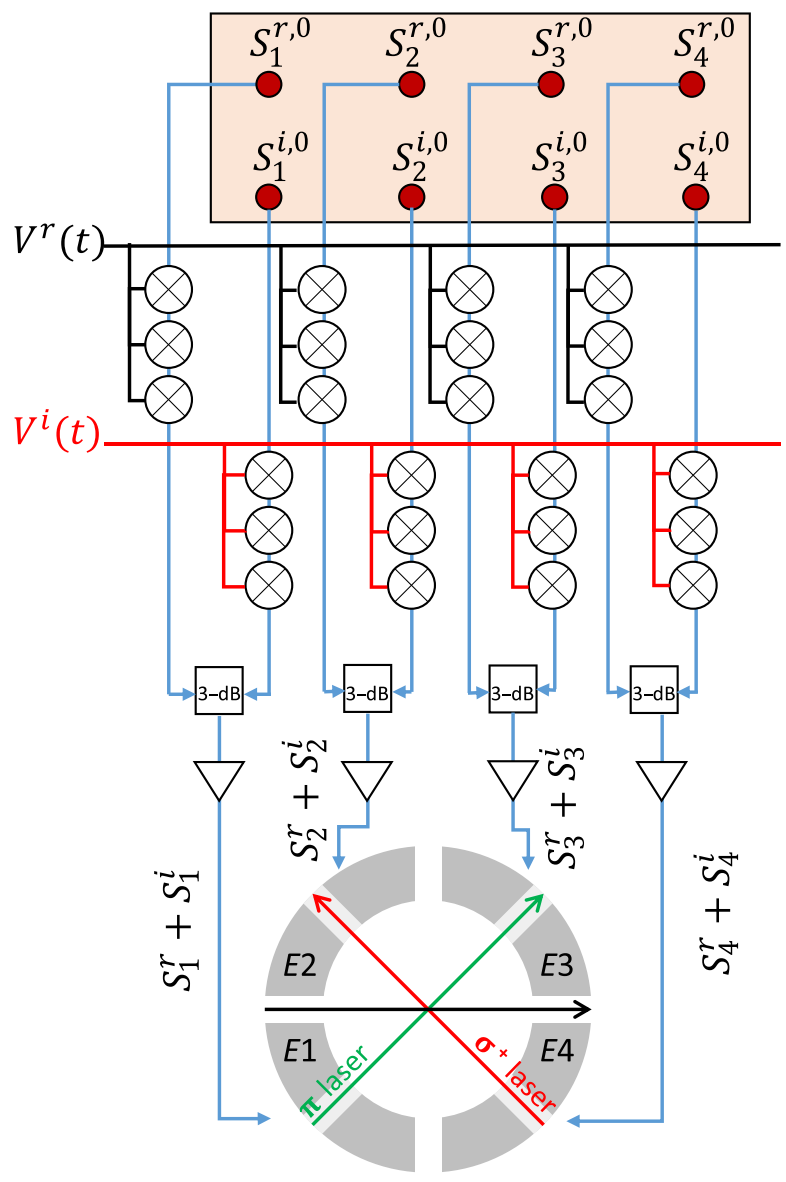

FIG. 11. Radio-frequency circuit. The outputs of an eightchannel synthesizer (light red), labeled $S_{1}^{r, 0}, S_{2}^{r, 0}, S_{3}^{r, 0}, S_{4}^{r, 0}, S_{1}^{i, 0}$, $S_{2}^{i, 0}, S_{3}^{i, 0}$, and $S_{4}^{i, 0}$, are sent to eight sets of three successive mixers that are controlled either by the voltage $V_{r}(t)$ (black line, for the outputs $S_{1}^{r, 0}, S_{2}^{r, 0}, S_{3}^{r, 0}, S_{4}^{r, 0}$ ) or by $V_{i}(t)$ (red line, for the outputs $\left.S_{1}^{i, 0}, S_{2}^{i, 0}, S_{3}^{i, 0}, S_{4}^{i, 0}\right)$. The resulting signals are combined two by two using $3-\mathrm{dB}$ couplers and sent to amplifiers, which feed the four ring electrodes surrounding the experiment (in gray).

used as voltage-controlled attenuators. Control voltages are generated by a two-channel arbitrary waveform generator with a $1 \mathrm{~ns}$ time resolution. (Fig. 11).

The field $\mathbf{F}_{\text {rf }}(t)$ is the sum of the two quadrature vector fields defined by

$$
\begin{aligned}
& \mathbf{F}_{r}(t)=F_{r}(t)\left[\cos \left(\omega_{\mathrm{rf}} t\right) \mathbf{u}_{x}+\sin \left(\omega_{\mathrm{rf}} t\right) \mathbf{u}_{y}\right], \\
& \mathbf{F}_{i}(t)=F_{i}(t)\left[-\sin \left(\omega_{\mathrm{rf}} t\right) \mathbf{u}_{x}+\cos \left(\omega_{\mathrm{rf}} t\right) \mathbf{u}_{y}\right] .
\end{aligned}
$$

In order to determine the conversion between $F_{r}(t)$ and $V_{r}(t)$ [or $F_{i}(t)$ and $V_{i}(t)$ ], we first record how the amplitudes of the rf signals after the amplifiers, $S_{k}^{r}$ and $S_{k}^{i}$, vary as we change the control voltages $V_{r}$ and $V_{i}$. Figure 12 presents the results of this measurement (normalized to the value of the amplitude for $\left.V_{r}=V_{i}=V_{0}=1.52 \mathrm{~V}\right)$. We observe that all channels behave quite similarly (up to a $\sim 10 \%$ deviation for channel 2 and large values of the drive voltage). Using the measurement of the $k=3$ channel, we define 


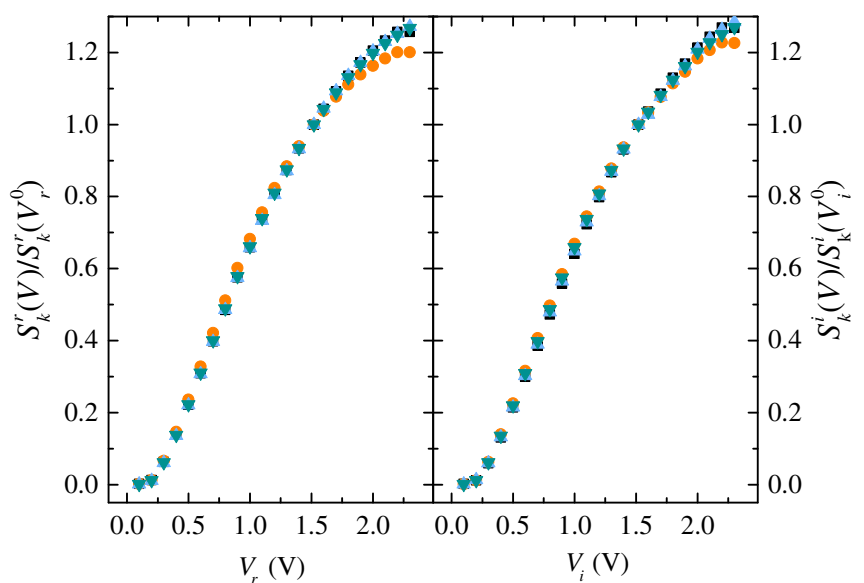

FIG. 12. dc response of the mixers. Left: Measurement of the ratio $S_{k}^{r}(V) / S_{k}^{r}\left(V_{r}^{0}\right)$, where $S_{k}^{r}(V)$ is the amplitude of the signal $S_{k}^{r}(t)$ when the voltage $V_{r}(t)$ is set to $V$ for $k=1$ (black squares), $k=2$ (orange circles), $k=3$ (blue upward triangles), and $k=4$ (green downward triangles). Right: Measurement of the ratio $S_{k}^{i}(V) / S_{k}^{i}\left(V_{i}^{0}\right)$, where $S_{k}^{i}(V)$ is the amplitude of the signal $S_{k}^{i}(t)$ when the voltage $V_{i}(t)$ is set to $V$ (same color code). We choose $k=3$ to determine $f_{r}(V)$ and $f_{i}(V)$.

$$
\begin{gathered}
f_{r}\left(V_{r}\right)=S_{3}^{r}\left(V_{r}\right) / S_{3}^{r}\left(V_{0}\right), \\
f_{i}\left(V_{i}\right)=S_{3}^{i}\left(V_{i}\right) / S_{3}^{i}\left(V_{0}\right) .
\end{gathered}
$$

We then use the atomic signal to measure the amplitude of the rf field $F_{r}^{0}$ corresponding to $V_{r}=V_{0}$. To that end, we prepare the atoms in the $52 c$ state and apply to the mixers a pulse $V_{r}(t)$ of constant amplitude $V_{0}$ and variable duration $\tau$. Such a rf pulse induces a rotation of the spin coherent state describing the atoms at an angular frequency $\Omega_{\mathrm{rf}}^{52}$, which is directly proportional to the amplitude $F_{r}^{0}$ of the rf field at the position of the atoms when $V_{r}(t)$ is equal to $V_{0}$. By measuring the population of the levels $52 c, 52 e_{1}$, and $52 e_{2}$ as a function of $\tau$, we infer $\Omega_{\mathrm{rf}}^{52}$ and measure $F_{r}^{0}=4.37 \pm 0.03 \mathrm{~V} / \mathrm{m}$. We measure with the same method the amplitude $F_{i}^{0}=4.53 \pm 0.02 \mathrm{~V} / \mathrm{m}$ of the $\mathrm{rf}$ field when $V_{i}(t)=V_{0}$.

We finally have

$$
\begin{aligned}
& F_{r}(t)=F_{r}^{0} f_{r}\left(V_{r}(t)\right), \\
& F_{i}(t)=F_{i}^{0} f_{i}\left(V_{i}(t)\right) .
\end{aligned}
$$

We invert these equations to convert the optimal pulse amplitude $F_{r}(t)$ and $F_{i}(t)$ into the time-dependent voltages $V_{r}(t)$ and $V_{i}(t)$. This method assumes that all mixers provide exactly the same attenuation, and neglects the frequency response of $f_{r}(V)$ and $f_{i}(V)$ as well as the nonlinearity of the amplifiers at the end of the rf generation chain. It thus only provides a rough calibration. After the conversion, we perform a final "closed loop" optimization of the shape of $V_{r}(t)$ and $V_{i}(t)$ on the atomic signal as described in Appendix F.

\section{APPENDIX F: EXPERIMENTAL OPTIMIZATION OF THE PULSES}

\section{Optimization of the $52 c$ preparation}

We optimize the first $40 \mathrm{~ns}$ of the pulse by globally rescaling the amplitude of the pulse by an adjustable factor $\lambda$. This provides a first-order compensation for the uncertainty on the determination of $f_{r}, f_{i}, F_{r}^{0}$, and $F_{i}^{0}$ and on the frequency response of the mixer. The last $73 \mathrm{~ns}$ of the pulse correspond to a rotation of the spin coherent state toward the north pole of the Bloch sphere. This part is very sensitive to phase and amplitude calibration of the rf. For a given value of $\lambda$, we directly optimize iteratively the amplitude of the plateaus of $V_{r}(t)$ and $V_{i}(t)$ on the population of $52 c$.

We repeat the optimization for different values of $\lambda$ between 0.9 and 1 . Figure 13 presents the populations $P_{c}$, $P_{e_{1}}$, and $P_{e_{2}}$ for each value of $\lambda$ once the plateaus have been optimized. The flat optimum shows that the optimized pulse is very robust to small changes. We finally choose the value $\lambda=0.95$, which has the highest $P_{c}$ and the lowest $P_{e_{2}}$.

\section{Optimization of coherence test rf pulse}

For demonstrating the coherence of the optimal circularization pulse, we start the sequence with a superposition of levels $52 c$ and $50 c$. We have to adapt the optimal pulse in order to additionally preserve the population of $50 \mathrm{c}$. In the static field $F_{0}$, the Stark frequency in the $n=50$ manifold is only $\Delta / 2 \pi=10 \mathrm{MHz}$ smaller than $\omega_{\mathrm{rf}} / 2 \pi$. Therefore, the rf pulse also affects the state of the atoms in the $n=50$ manifold. The first $40 \mathrm{~ns}$ of the optimal pulse transfer $50 \mathrm{c}$ into a spin coherent state $|\theta, \phi\rangle_{50}$. We optimize the last

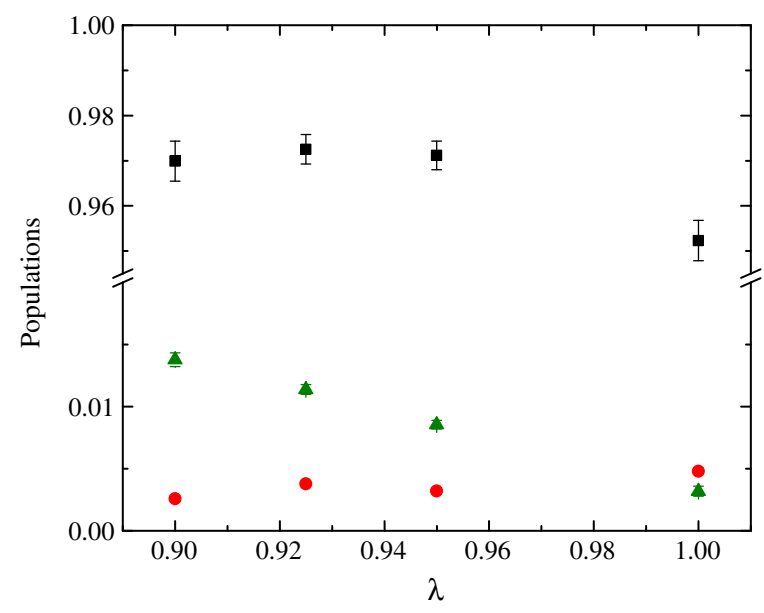

FIG. 13. Scaling factor optimization. Populations of the $52 c$ (black), $52 e_{1}$ (red), and $52 e_{2}$ (green) states obtained after optimizing the parameter of the rotation part of the circularization pulse, for different values of the scaling factor $\lambda$. 
73 ns of the pulse so that they induce a rotation that maps $|\theta, \phi\rangle_{50}$ into $50 c$.

In the frame rotating at frequency $\omega_{\mathrm{rf}} / 2 \pi$, a rf field of fixed phase and amplitude induces a rotation of the effective spin defined by the rotation vector,

$$
\tau \boldsymbol{\Omega}^{52}=\tau\left(\begin{array}{c}
\Omega_{\mathrm{rf}}^{52} \cos \phi \\
\Omega_{\mathrm{rf}}^{52} \sin \phi \\
0
\end{array}\right),
$$

where $\phi$ is the phase of the pulse and $\Omega_{\mathrm{rf}}^{52}$ the Rabi frequency, proportional to the $\mathrm{rf}$ amplitude. In the $n=50$ manifold, due to the detuning $\Delta$, the same pulse induces a rotation of the effective spin defined by

$$
\tau \boldsymbol{\Omega}^{50}=\tau\left(\begin{array}{c}
\Omega_{\mathrm{rf}}^{50} \cos \phi \\
\Omega_{\mathrm{rf}}^{50} \sin \phi \\
\Delta
\end{array}\right),
$$

where $\Omega_{\mathrm{rf}}^{50}=(50 / 52) \Omega_{\mathrm{rf}}^{52}$.

To ensure that $|\theta, \phi\rangle_{50}$ is rotated to $50 c$ at the end of the pulse, we replace the single $73 \mathrm{~ns}$ pulse of constant amplitude (phase $\phi$, Rabi frequency $\Omega_{\mathrm{rf}, 0}^{52}$ ) by two pulses of duration $\tau_{1}$ and $\tau_{2}$, with same phase $\phi$ but different amplitudes $\Omega_{\mathrm{rf}, 1}^{52}$ and $\Omega_{\mathrm{rf}, 2}^{52}$, such that

$$
\begin{aligned}
\tau_{1}+\tau_{2} & =73 \mathrm{~ns}, \\
\tau_{1} \Omega_{\mathrm{rf}, 1}^{52}+\tau_{2} \Omega_{\mathrm{rf}, 2}^{52} & =\left(\tau_{1}+\tau_{2}\right) \Omega_{\mathrm{rf}, 0}^{52} .
\end{aligned}
$$

The condition (F1) ensures that the two-amplitude pulse induces in the $n=52$ manifold the same rotation as the constant amplitude pulse. By tuning the ratio between $\Omega_{\mathrm{rf}, 1}^{52}$ and $\Omega_{\mathrm{rf}, 2}^{52}$ and $\tau_{1}$ and $\tau_{2}$, it is possible to ensure at the same time that the part of the wave function in the $n=50$ manifold returns into $50 c$ at the end of the pulse. We optimize experimentally $\left\{\Omega_{\mathrm{rf}, 1}^{52}, \Omega_{\mathrm{rf}, 2}^{52}, \tau_{1}, \tau_{2}\right\}$, with the constraint (F1) until the pulse transfers $50 \mathrm{c}$ into $50 \mathrm{c}$ [measured efficiency 98.2(5)\%].

We implement the time reversal of the preparation pulse [first $113 \mathrm{~ns}$ of Fig. 3(a) in the main paper] by inverting the amplitude of one of the quadratures and programming the pulse backward in time. Experimentally, we fine-tune again the values of $\left\{\Omega_{\mathrm{rf}, 1}^{\prime 52}, \Omega_{\mathrm{rf}, 2}^{\prime 52}, \tau_{1}^{\prime}, \tau_{2}^{\prime}\right\}$ of the time-reversed pulse to ensure that it transfers $50 \mathrm{c}$ into $50 \mathrm{c}$ [measured efficiency $98.2(8) \%$ ] and $52 c$ into $52 m_{2}$ [measured efficiency $88.1(9) \%]$.

\section{Optimization of the pulse preparing the superposition $52 m_{1}$ and $52 c$}

The first part of the pulse produces a superposition of a spin coherent state and of the rf dressed state that adiabatically evolves into $52 m_{1}$ when the rf is turned off. The second part of the pulse brings the spin coherent state to $52 c$, and slowly switches off so that population of the dressed state ends up in $52 m_{1}$.

The optimization process is similar to that of the circularization pulse. First, we globally rescale the first $80 \mathrm{~ns}$ of the pulse with a scaling factor (leading to $\lambda=0.925)$. Then, we adjust the amplitude of the two quadratures during the last part of the pulse to optimize the transfer of the spin coherent state into the circular state. To ensure that the other part of the wave function adiabatically follows the instantaneous dressed state of the rf, we vary linearly $V_{r}(t)$ and $V_{i}(t)$ between $t=80 \mathrm{~ns}, t=t_{1}=$ $136 \mathrm{~ns}$, and $t=167 \mathrm{~ns}$. By varying the values of $V_{r}\left(t_{1}\right)$ and $V_{i}\left(t_{1}\right)$ characteristic of the linear ramps, we optimize the probability to end up in $52 c$, without affecting the probability to end up in $52 m_{1}$ at the end of the pulse. Note that the final ramp is slightly longer than in the simulation to compensate for the shape of $f_{r}$ and $f_{i}$ (Fig. 12): the rf field amplitudes $F_{r}(t)$ and $F_{i}(t)$ go to zero faster than the amplitude of $V_{r}(t)$ and $V_{i}(t)$.

However, this optimization was not sufficient to obtain a perfect balance between $52 c$ and $52 m_{1}$. We found that the final population of $52 m_{1}$ is very sensitive to the amplitude of the optimal pulse during its first $4 \mathrm{~ns}$. At this timescale, we can no longer correct the frequency response of the mixer by a global scaling factor $\lambda$. We need to rescale independently the amplitude of the driving voltage $\left[V_{r}(t) \rightarrow\right.$ $\alpha V_{r}(t)$ and $\left.V_{i}(t) \rightarrow \alpha V_{i}(t)\right]$ for $t<4$ ns. Figure 14 presents the final population of $52 m_{1}$ as a function of the scaling factor $\alpha$. For $\alpha=2.8$, we obtain a final population of $P_{m_{1}}=0.502(6)$.

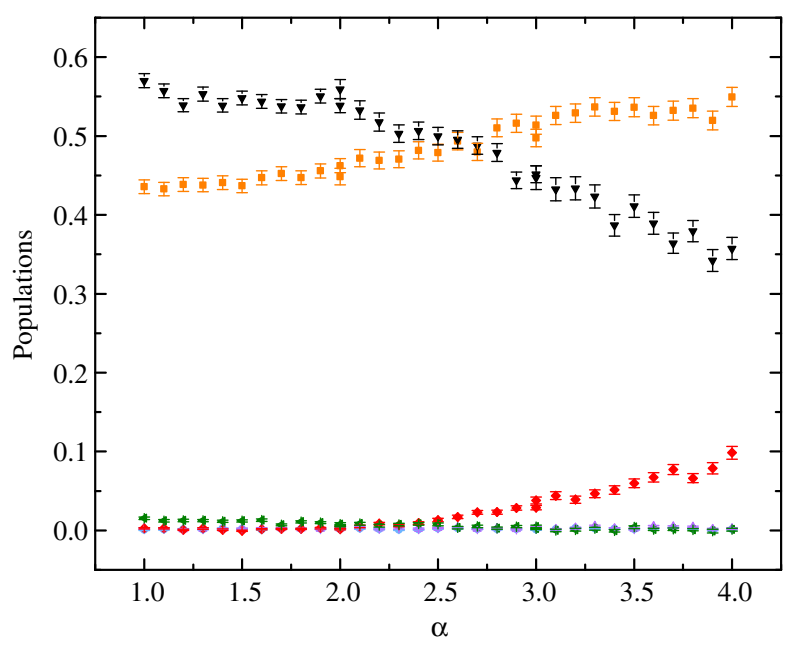

FIG. 14. Optimization of the first lobe of the cat state pulse. Populations of the level $m_{1}, m_{2}, m_{3}, e_{2}, e_{1}$, and $c$ as a function of the scaling factor $\alpha$ with which we amplify the first 4 ns of the pulse. Increasing $\alpha$ increases the final population of $52 m_{1}$ and decrease the final population of $52 c$ (until eventually the SCS part of the wave function no longer reaches the north pole at the end of the pulse, leading to an increase of $P_{e_{1}}$ ). 


\section{Optimization of the time-reversed pulse recombining the $52 m_{1}$ and $52 c$}

To measure the coherence of the superposition of $52 m_{1}$ and $52 c$, we program the time reversal of the pulse of Fig. 4(a). In principle, as the pulse of Fig. 4(a) performs the transfer

$$
\left|52 m_{2}\right\rangle \rightarrow \frac{1}{\sqrt{2}}\left(\left|52 m_{1}\right\rangle-|52 c\rangle\right)
$$

and the evolution is unitary, the time-reversed pulse must perform the transfer

$$
\begin{aligned}
|52 c\rangle & \rightarrow \frac{1}{\sqrt{2}}\left(-\left|52 m_{2}\right\rangle+\left|52 m_{2}\right\rangle^{\perp}\right), \\
\left|52 m_{1}\right\rangle & \rightarrow \frac{1}{\sqrt{2}}\left(\left|52 m_{2}\right\rangle+\left|52 m_{2}\right\rangle^{\perp}\right),
\end{aligned}
$$

where $\left|52 m_{2}\right\rangle^{\perp}$ is a state orthogonal to $\left|52 m_{2}\right\rangle$. Therefore, as we vary the relative phase of the superposition of $|52 c\rangle$ and $\left|52 m_{1}\right\rangle$ before applying the time-reversed pulse, one should observe constructive and destructive interferences in the amplitude of probability to end up in $\left|52 m_{2}\right\rangle$. The visibility of this interference pattern gives the degree of coherence of the superposition.

Experimentally, we find that if we simply program $V_{r}(t) \rightarrow-V_{r}(-t)$ and $V_{i}(t) \rightarrow V_{i}(-t)$, the resulting pulse transfers the state $52 c$ into $52 m_{2}$ with a probability $p_{c \rightarrow 2}=$ $0.61(2)$ and the state $52 m_{1}$ into $52 m_{2}$ with a probability $p_{1 \rightarrow 2}=0.28(1)$. This would lead to a strong limitation of the visibility of the interference. The remaining population is found to be in $52 m_{1}\left[p_{c \rightarrow 1}=0.25(1)\right.$ for $52 c$ and in $p_{1 \rightarrow 1}=0.56(2)$ for $\left.52 m_{1}\right]$. To optimize the contrast of the fringes, we add after the time-reversed pulse an additional rf pulse, tuned on the $52 m_{1}-52 m_{2}$ transition, whose phase is chosen to decrease $p_{c \rightarrow 2}$ and increase $p_{1 \rightarrow 2}$. With this additional pulse, we obtain $p_{c \rightarrow 2}^{\text {final }}=p_{1 \rightarrow 2}^{\text {final }} \approx 0.44$.

\section{APPENDIX G: ELECTRIC FIELD NOISE AND EXTRAPOLATION TO ZERO FIELD}

Because of the differential Stark effect between the levels $52 m_{2}, 52 m_{1}$, and $52 c$, the visibility of the fringes of Figs. 3(b) and 4(b) is very sensitive to the electric field noise. To quantify this effect, we vary the time delay between the two rf pulses that prepare and recombine the "cat states" $\left(|50 c\rangle+\left|52 m_{2}\right\rangle\right.$ or $|52 c\rangle-\left|52 m_{1}\right\rangle$, respectively) and measure, for each delay, the visibility of the fringes. The longer the atoms stay in the superposition, the more sensitive the relative phase is to the electric field, reducing the visibility of the fringes. To calibrate the sensitivity of the relative phase to the electric field, we record the fringes for two slightly different values of the static electric field [4]. Figure 15 shows the visibility as a function of the phase sensitivity $\xi[\mathrm{in} \mathrm{rad} /(\mathrm{mV} / \mathrm{m})]$ for the

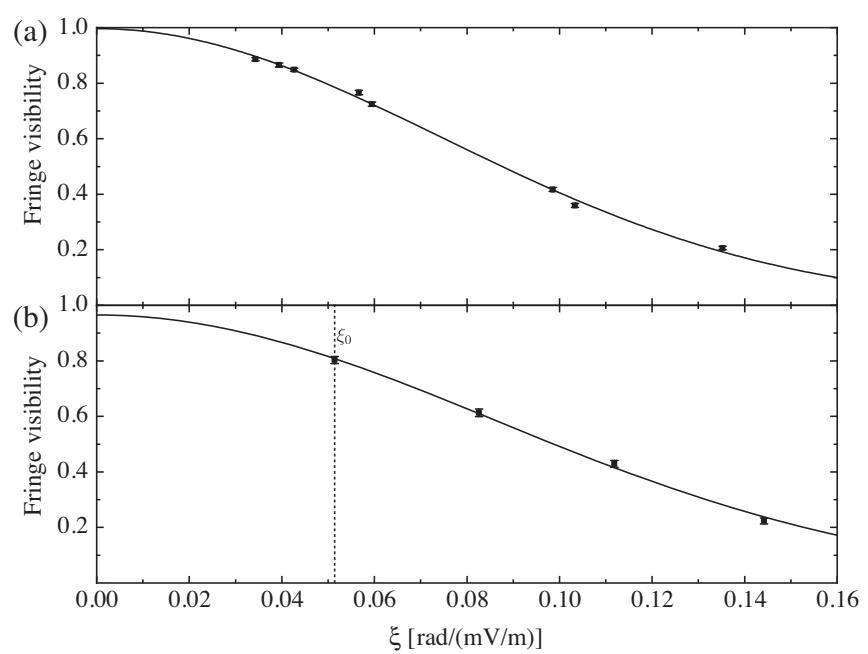

FIG. 15. Visibility as a function of the field sensitivity. Visibility of the fringes measuring the coherence of (a) $|50 c\rangle+$ $\left|52 m_{2}\right\rangle$ and (b) $|52 c\rangle+\left|52 m_{1}\right\rangle$, as function of the phase sensitivity of the fringes to the electric field $\xi$. The points are experimental (with statistical error bars), the line is a Gaussian fit of the form $V=\mathcal{V}_{0} \exp \left(-\xi^{2} \sigma_{F}^{2} / 2\right)$, where $\mathcal{V}_{0}$ is the intrinsic visibility that the fringes would have in the absence of electric field noise.

two superpositions. Assuming a Gaussian electric field noise, we expect the visibility $\mathcal{V}$ to be

$$
\mathcal{V}=\mathcal{V}_{0} e^{-(1 / 2) \xi^{2} \sigma_{F}^{2}},
$$

where $\sigma_{F}$ is the variance of the electric field noise and $\mathcal{V}_{0}$ the intrinsic visibility in the absence of noise. A fit to the experimental data gives $\mathcal{V}_{0}=1.00(1)$ [and $\sigma_{F}=$ $13.4(1) \mathrm{mV} / \mathrm{m}]$ for the superposition $|50 c\rangle+\left|52 m_{2}\right\rangle$. For the superposition $|52 c\rangle+\left|52 m_{1}\right\rangle$, we find $\mathcal{V}_{0}=0.97(2)$ and a slightly cleaner field $\sigma_{F}=11.6(1) \mathrm{mV} / \mathrm{m}$ (the two sets of data have been recorded at a few months time interval).

By symmetry, the phase sensitivity of the preparation of the superposition is half that of the full process of preparation and recombination. As a result, the degree of coherence $C$ of the superposition $|52 c\rangle-\left|52 m_{1}\right\rangle$ after the rf pulse is

$$
C \geq \mathcal{V}_{0} e^{-(1 / 2)\left(\xi_{0} / 2\right)^{2} \sigma_{F}^{2}} \approx 93 \%
$$

where $\xi_{0}$ is the phase sensitivity for the shortest $\Delta t$. The final fidelity $\mathcal{F}$ is then

$$
\mathcal{F}=\frac{1}{2}\left(P_{c}+P_{m_{1}}+2 C \sqrt{P_{c} P_{m_{1}}}\right) \geq 0.93 .
$$

[1] M. A. Nielsen and I. L. Chuang, Quantum Computation and Quantum Information (Cambridge University Press, Cambridge, England, 2000). 
[2] D. P. DiVincenzo, The Physical Implementation of Quantum Computation, Fortschr. Phys. 48, 771 (2000).

[3] J. Bourassa, F. Beaudoin, J. M. Gambetta, and A. Blais, Josephson-Junction-Embedded Transmission-Line Resonators: From Kerr Medium to In-Line Transmon, Phys. Rev. A 86, 013814 (2012).

[4] A. Facon, E.-K. Dietsche, D. Grosso, S. Haroche, J.-M. Raimond, M. Brune, and S. Gleyzes, A Sensitive Electrometer Based on a Rydberg Atom in a Schrödinger-Cat State, Nature (London) 535, 262 (2016).

[5] F. Dolde, V. Bergholm, Y. Wang, I. Jakobi, B. Naydenov, S. Pezzagna, J. Meijer, F. Jelezko, P. Neumann, T. SchulteHerbrüggen et al., High-Fidelity Spin Entanglement Using Optimal Control, Nat. Commun. 5, 3371 (2014).

[6] R. Blatt and C. F. Roos, Quantum Simulations with Trapped Ions, Nat. Phys. 8, 277 (2012).

[7] A. Omran, H. Levine, A. Keesling, G. Semeghini, T. T. Wang, S. Ebadi, H. Bernien, A. S. Zibrov, H. Pichler, S. Choi, J. Cui, M. Rossignolo, P. Rembold, S. Montangero, T. Calarco, M. Endres, M. Greiner, V. Vuletić, and M. D. Lukin, Generation and Manipulation of Schrödinger Cat States in Rydberg Atom Arrays, Science 365, 570 (2019).

[8] S. J. Glaser, U. Boscain, T. Calarco, C. P. Koch, W. Köckenberger, R. Kosloff, I. Kuprov, B. Luy, S. Schirmer, T. Schulte-Herbrüggen, D. Sugny, and F. K. Wilhelm, Training Schrödinger's Cat: Quantum Optimal Control. Strategic Report on Current Status, Visions and Goals for Research in Europe, Eur. Phys. J. D 69, 279 (2015).

[9] C. P. Koch, Controlling Open Quantum Systems: Tools, Achievements, and Limitations, J. Phys. Condens. Matter 28, 213001 (2016).

[10] T. Keating, C. H. Baldwin, Y.-Y. Jau, J. Lee, G. W. Biedermann, and I. H. Deutsch, Arbitrary Dicke-State Control of Symmetric Rydberg Ensembles, Phys. Rev. Lett. 117, 213601 (2016).

[11] K. Singer, U. Poschinger, M. Murphy, P. Ivanov, F. Ziesel, T. Calarco, and F. Schmidt-Kaler, Colloquium: Trapped Ions as Quantum Bits: Essential Numerical Tools, Rev. Mod. Phys. 82, 2609 (2010).

[12] A. V. Gorshkov, T. Calarco, M. D. Lukin, and A. S. Sørensen, Photon Storage in $\Lambda$-Type Optically Dense Atomic Media. IV. Optimal Control Using Gradient Ascent, Phys. Rev. A 77, 043806 (2008).

[13] L. Giannelli, T. Schmit, T. Calarco, C. P. Koch, S. Ritter, and G. Morigi, Optimal Storage of a Single Photon by a Single Intra-Cavity Atom, New J. Phys. 20, 105009 (2018).

[14] M. Lapert, Y. Zhang, M. Braun, S. J. Glaser, and D. Sugny, Singular Extremals for the Time-Optimal Control of Dissipative Spin $\frac{1}{2}$ Particles, Phys. Rev. Lett. 104, 083001 (2010).

[15] F. Poggiali, P. Cappellaro, and N. Fabbri, Optimal Control for One-Qubit Quantum Sensing, Phys. Rev. X 8, 021059 (2018).

[16] S. Montangero, T. Calarco, and R. Fazio, Robust Optimal Quantum Gates for Josephson Charge Qubits, Phys. Rev. Lett. 99, 170501 (2007).

[17] D. J. Egger and F. K. Wilhelm, Adaptive Hybrid Optimal Quantum Control for Imprecisely Characterized Systems, Phys. Rev. Lett. 112, 240503 (2014).
[18] T. Häberle, D. Schmid-Lorch, K. Karrai, F. Reinhard, and J. Wrachtrup, High-Dynamic-Range Imaging of Nanoscale Magnetic Fields Using Optimal Control of a Single Qubit, Phys. Rev. Lett. 111, 170801 (2013).

[19] G. Waldherr, Y. Wang, S. Zaiser, M. Jamali, T. SchulteHerbrüggen, H. Abe, T. Ohshima, J. Isoya, J. F. Du, P. Neumann, and J. Wrachtrup, Quantum Error Correction in a Solid-State Hybrid Spin Register, Nature (London) 506, 204 (2014).

[20] T. H. Taminiau, J. Cramer, T. van der Sar, V. V. Dobrovitski, and R. Hanson, Universal Control and Error Correction in Multi-Qubit Spin Registers in Diamond, Nat. Nanotechnol. 9, 171 (2014).

[21] T. Nöbauer, A. Angerer, B. Bartels, M. Trupke, S. Rotter, J. Schmiedmayer, F. Mintert, and J. Majer, Smooth Optimal Quantum Control for Robust SolidState Spin Magnetometry, Phys. Rev. Lett. 115, 190801 (2015).

[22] B. E. Anderson, H. Sosa-Martinez, C. A. Riofrío, I. H. Deutsch, and P.S. Jessen, Accurate and Robust Unitary Transformations of a High-Dimensional Quantum System, Phys. Rev. Lett. 114, 240401 (2015).

[23] S. van Frank, A. Negretti, T. Berrada, R. Bücker, S. Montangero, J.-F. Schaff, T. Schumm, T. Calarco, and J. Schmiedmayer, Interferometry with Non-Classical Motional States of a Bose-Einstein Condensate, Nat. Commun. 5, 4009 (2014).

[24] N. Ofek, A. Petrenko, R. Heeres, P. Reinhold, Z. Leghtas, B. Vlastakis, Y. Liu, L. Frunzio, S. M. Girvin, L. Jiang, M. Mirrahimi, M. H. Devoret, and R. J. Schoelkopf, Extending the Lifetime of a Quantum Bit with Error Correction in Superconducting Circuits, Nature (London) 536, 441 (2016).

[25] S. van Frank, M. Bonneau, J. Schmiedmayer, S. Hild, C. Gross, M. Cheneau, I. Bloch, T. Pichler, A. Negretti, T. Calarco et al., Optimal Control of Complex Atomic Quantum Systems, Sci. Rep. 6, 34187 (2016).

[26] R. W. Heeres, P. Reinhold, N. Ofek, L. Frunzio, L. Jiang, M. H. Devoret, and R. J. Schoelkopf, Implementing a Universal Gate Set on a Logical Qubit Encoded in an Oscillator, Nat. Commun. 8, 94 (2017).

[27] Z. Leghtas, G. Kirchmair, B. Vlastakis, M. H. Devoret, R. J. Schoelkopf, and M. Mirrahimi, Deterministic Protocol for Mapping a Qubit to Coherent State Superpositions in a Cavity, Phys. Rev. A 87, 042315 (2013).

[28] M. Saffman, T. G. Walker, and K. Mølmer, Quantum Information with Rydberg Atoms, Rev. Mod. Phys. 82, 2313 (2010).

[29] C. Sayrin, I. Dotsenko, X. Zhou, B. Peaudecerf, T. Rybarczyk, S. Gleyzes, P. Rouchon, M. Mirrahimi, H. Amini, M. Brune, J.-M. Raimond, and S. Haroche, Real-Time Quantum Feedback Prepares and Stabilizes Photon Number States, Nature (London) 477, 73 (2011).

[30] T. Xia, X. L. Zhang, and M. Saffman, Analysis of a Controlled Phase Gate Using Circular Rydberg States, Phys. Rev. A 88, 062337 (2013).

[31] M. Saffman, Quantum Computing with Atomic Qubits and Rydberg Interactions: Progress and Challenges, J. Phys. B 49, 202001 (2016). 
[32] R. W. Andrews, R. W. Peterson, T. P. Purdy, K. Cicak, R. W. Simmonds, C. A. Regal, and K. W. Lehnert, Bidirectional and Efficient Conversion between Microwave and Optical Light, Nat. Phys. 10, 321 (2014).

[33] A. Signoles, E. K. Dietsche, A. Facon, D. Grosso, S. Haroche, J. M. Raimond, M. Brune, and S. Gleyzes, Coherent Transfer between Low-Angular-Momentum and Circular Rydberg States, Phys. Rev. Lett. 118, 253603 (2017).

[34] E. K. Dietsche, A. Larrouy, S. Haroche, J. M. Raimond, M. Brune, and S. Gleyzes, High-Sensitivity Magnetometry with a Single Atom in a Superposition of Two Circular Rydberg States, Nat. Phys. 15, 326 (2019).

[35] S. Patsch, D. M. Reich, J.-M. Raimond, M. Brune, S. Gleyzes, and C. P. Koch, Fast and Accurate Circularization of a Rydberg Atom, Phys. Rev. A 97, 053418 (2018).

[36] T.F. Gallagher, Rydberg Atoms (Cambridge University Press, Cambridge, England, 1994).
[37] M. L. Zimmerman, M. G. Littman, M. M. Kash, and D. Kleppner, Stark Structure of the Rydberg States of AlkaliMetal Atoms, Phys. Rev. A 20, 2251 (1979).

[38] D. M. Reich, M. Ndong, and C. P. Koch, Monotonically Convergent Optimization in Quantum Control Using Krotov's Method, J. Chem. Phys. 136, 104103 (2012).

[39] M. Goerz, D. Basilewitsch, F. Gago-Encinas, M. G. Krauss, K. P. Horn, D. M. Reich, and C. Koch, Krotov: A Python Implementation of Krotov's Method for Quantum Optimal Control, SciPost Phys. 7, 080 (2019).

[40] E. Dietsche, Quantum Sensing with Rydberg Schrödinger Cat States, Ph. D. thesis, Université Pierre et Marie CurieParis, 2017, https://tel.archives-ouvertes.fr/tel-01735459.

[41] A. Signoles, A. Facon, D. Grosso, I. Dotsenko, S. Haroche, J.-M. Raimond, M. Brune, and S. Gleyzes, Confined Quantum Zeno Dynamics of a Watched Atomic Arrow, Nat. Phys. 10, 715 (2014). 